\title{
Approximation of Functions on a Square by Interpolation Polynomials at Vertices and Few Fourier Coefficients
}

\author{
Zhihua Zhang ${ }^{1,2}$ \\ ${ }^{1}$ College of Global Change and Earth System Science, Beijing Normal University, Beijing 100875, China \\ ${ }^{2}$ Joint Centre for Global Change Studies, Beijing, China \\ Correspondence should be addressed to Zhihua Zhang; zhangzh@bnu.edu.cn
}

Received 9 December 2016; Accepted 19 March 2017; Published 22 May 2017

Academic Editor: Hua Su

Copyright (c) 2017 Zhihua Zhang. This is an open access article distributed under the Creative Commons Attribution License, which permits unrestricted use, distribution, and reproduction in any medium, provided the original work is properly cited.

For a bivariate function on a square, in general, its Fourier coefficients decay slowly, so one cannot reconstruct it by few Fourier coefficients. In this paper we will develop a new approximation scheme to overcome the weakness of Fourier approximation. In detail, we will use Lagrange interpolation and linear interpolation on the boundary of the square to derive a new approximation scheme such that we can use the values of the target function at vertices of the square and few Fourier coefficients to reconstruct the target function with very small error.

\section{Introduction}

It is well-known that smooth periodic functions can be approximated well by Fourier series $[1,2]$. But, if we expand a bivariate function on the square robustly into Fourier series, in general, its Fourier coefficients decay slowly. Therefore, one cannot reconstruct it by few Fourier coefficients [3-5]. This is also viewed as the weakness of Fourier approximation compared with wavelet approximation [6,7]. In this paper we will develop a new approximation scheme to overcome the weakness of Fourier approximation. Our main idea is as follows: For a smooth function on the square $[0,1]^{2}$, based on the Lagrange interpolation and the linear interpolation [8-11] of the target function $f$ at the vertices of $[0,1]^{2}$, we construct a bivariate function $g$ such that $f=g$ on the boundary of $[0,1]^{2}$. Secondly, we take Fourier expansion of the residual $r=f-g$. Finally, based on this decomposition, we can derive a new scheme to approximate $f$ by using the values of $f$ at the vertices of $[0,1]^{2}$ and few Fourier coefficients.

Throughout this paper we denote the boundary of the unit square $[0,1]^{2}$ by $\partial\left([0,1]^{2}\right)$ and denote the vertices of $[0,1]^{2}$ by $\{0,1\}^{2}$; that is,

$$
\{0,1\}^{2}=\{(0,0),(0,1),(1,0),(1,1)\} .
$$

Denote $f_{x^{i} y^{j}}=\partial^{i+j} f / \partial x^{i} \partial y^{j}$ and $\sum_{k}=\sum_{k=-\infty}^{\infty}$. We say $f \epsilon$ $C^{(3,3)}\left([0,1]^{2}\right)$ if $f_{x^{3} y^{3}} \in C\left([0,1]^{2}\right)$.

Expand $f$ into a Fourier series:

$$
f(x, y)=\sum_{m} \sum_{n} c_{m n}(f) e^{2 \pi i(m x+n y)},
$$

where $c_{m n}(f)$ is the Fourier coefficient of $f$ :

$$
c_{m n}(f)=\iint_{0}^{1} f(x, y) e^{-2 \pi i(m x+n y)} \mathrm{d} x \mathrm{~d} y .
$$

The Fourier series hyperbolic cross truncations are defined as [12]

$$
s_{N}^{(h)}(f ; x, y)=\sum_{\substack{1 \leq|m| n|<N\\| m|,| n \mid<N}} c_{m n}(f) e^{2 \pi i(m x+n y)} .
$$

\section{New Approximation Scheme of Bivariate Functions}

Let $f(x, y)$ be defined on the square $[0,1]^{2}$. We use its values at vertices of the square $[0,1]^{2}$ and its few Fourier coefficients to reconstruct $f(x, y)$ on $[0,1]^{2}$. 
Step 1. Take the linear interpolation of $f$ on the vertical intercept $\{(x, y), 0 \leq y \leq 1\}$ of the square $[0,1]^{2}$, for a fixed $x(0 \leq x \leq 1)$ :

$$
g_{1}(x, y)=f(x, 0)(1-y)+f(x, 1) y \quad(0 \leq y \leq 1) .
$$

Let $\varphi^{(0)}(x)=f(x, 0)-(f(0,0)(1-x)+f(1,0) x)$. Expand $\varphi^{(0)}$ into Fourier series:

$$
\varphi^{(0)}(x)=\sum_{m} c_{m}\left(\varphi^{(0)}\right) e^{2 \pi i m x}
$$

where $c_{m}\left(\varphi^{(0)}\right)$ is the Fourier coefficient of $\varphi^{(0)}$. We reconstruct $f(x, 0)$ by

$$
\begin{aligned}
h_{N}^{(1)}(x)= & f(0,0)(1-x)+f(1,0) x \\
& +\sum_{|m|<N} c_{m}\left(\varphi^{(0)}\right) e^{2 \pi i m x} .
\end{aligned}
$$

Let $\varphi^{(1)}(x)=f(x, 1)-(f(0,0)(1-x)+f(1,1) x)$. Expand $\varphi^{(1)}$ into Fourier series:

$$
\varphi^{(1)}(x)=\sum_{m} c_{m}\left(\varphi^{(1)}\right) e^{2 \pi i m x}
$$

We reconstruct $f(x, 1)$ by

$$
\begin{aligned}
h_{N}^{(2)}(x)= & f(0,1)(1-x)+f(1,1) x \\
& +\sum_{|m|<N} c_{m}\left(\varphi^{(1)}\right) e^{2 \pi i m x} .
\end{aligned}
$$

So we reconstruct $g_{1}(x, y)$ by

$$
g_{1 N}(x, y)=h_{N}^{(1)}(x)(1-y)+h_{N}^{(2)}(x) y,
$$

that is, the values of $f$ at vertices $\{0,1\}^{2}$ and Fourier coefficients $c_{m}\left(\varphi^{(0)}\right), c_{m}\left(\varphi^{(1)}\right)(|m|<N)$.

Step 2. Take the linear interpolation of $f$ on the horizontal intercept $\{(x, y), 0 \leq x \leq 1\}$ of the square $[0,1]^{2}$, for a fixed $y(0 \leq y \leq 1)$

$$
g_{2}(x, y)=f(0, y)(1-x)+f(1, y) x \quad(0 \leq x \leq 1) .
$$

Let $\psi^{(0)}(y)=f(0, y)-(f(0,0)(1-y)+f(0,1) y)$. Expand $\psi^{(0)}$ into Fourier series:

$$
\psi^{(0)}(y)=\sum_{n} c_{n}\left(\psi^{(0)}\right) e^{2 \pi i n y}
$$

where $c_{n}\left(\psi^{(0)}\right)$ is the Fourier coefficient of $\psi^{(0)}$. We reconstruct $f(0, y)$ by

$$
\begin{aligned}
h_{N}^{(3)}(y)= & f(0,0)(1-y)+f(0,1) y \\
& +\sum_{|n|<N} c_{n}\left(\psi^{(0)}\right) e^{2 \pi i n y} .
\end{aligned}
$$

Let $\psi^{(1)}=f(1, y)-(f(1,0)(1-y)+f(1,1) y)$. Expand $\psi^{(1)}$ into Fourier series:

$$
\psi^{(1)}(y)=\sum_{n} c_{n}\left(\psi^{(1)}\right) e^{2 \pi i n y} .
$$

We reconstruct $f(1, y)$ by

$$
\begin{aligned}
h_{N}^{(4)}(y)= & f(1,0)(1-y)+f(1,1) y \\
& +\sum_{|n|<N} c_{n}\left(\psi^{(1)}\right) e^{2 \pi i n y} .
\end{aligned}
$$

So we reconstruct $g_{2}(x, y)$ by

$$
g_{2 N}(x, y)=h_{N}^{(3)}(y)(1-x)+h_{N}^{(4)}(y) x,
$$

that is, the values of $f$ at vertices $\{0,1\}^{2}$ and Fourier coefficients $c_{n}\left(\psi^{(0)}\right), c_{n}\left(\psi^{(1)}\right)(|n|<N)$.

Step 3. Take the bivariate interpolation polynomial of $f(x, y)$ with nodes $\{0,1\}^{2}$ :

$$
\begin{aligned}
g_{3}(x, y)= & f(0,0)(1-x)(1-y)+f(0,1)(1-x) y \\
& +f(1,0) x(1-y)+f(1,1) x y .
\end{aligned}
$$

Define an algebraic sum as

$$
\begin{array}{r}
g(x, y)=g_{1}(x, y)+g_{2}(x, y)-g_{3}(x, y) \\
\quad(0 \leq x, y \leq 1)
\end{array}
$$

which can be reconstructed by $g_{N}(x, y)=g_{1 N}(x, y)+$ $g_{2 N}(x, y)-g_{3}(x, y)$.

Step 4. Let

$$
r(x, y)=f(x, y)-g(x, y) .
$$

Expand $r(x, y)$ into Fourier series:

$$
r(x, y)=\sum_{m} \sum_{n} c_{m n}(r) e^{2 \pi i(m x+n y)} .
$$

Take the hyperbolic cross truncation of the Fourier series:

$$
r_{N}(x, y)=\sum_{\substack{1 \leq|m n|<N \\|m|,|n|<N}} c_{m n}(r) e^{2 \pi i(m x+n y)}
$$

which can reconstruct $r(x, y)$ by $r_{N}(x, y)$, that is, by few Fourier coefficients. Precisely say that the number $N_{d}$ of Fourier coefficients used in $r_{N}(x, y)$ is equal to $N \log N$; that is,

$$
N_{d} \sim N \log N \text {. }
$$

Step 5. We reconstruct the target function $f$ by

$$
f_{N}(x, y)=g_{N}(x, y)+r_{N}(x, y) \text {. }
$$

Now we can reconstruct a target function $f$ by the values of $f$ at $\{0,1\}^{2}: f(0,0), f(0,1), f(1,0), f(1,1)$ and Fourier coefficients $c_{m}\left(\varphi^{(0)}\right), c_{m}\left(\varphi^{(1)}\right)(|m|<N), c_{n}\left(\psi^{(0)}\right)$, $c_{n}\left(\psi^{(1)}\right)(|n|<N)$, and $\left\{c_{m n}(r)\right\}(|m n|<N,|m|,|n|<N)$. 


\section{Asymptotic Formula of the Errors for the Reconstruction of $g(x, y)$}

In order to give the error estimate of our approximation scheme, we need to first give the asymptotic formula of the Fourier coefficients.

Assume that $f \in C^{(3,3)}\left([0,1]^{2}\right)$. In Section 2, we know that $f(x, y)=g(x, y)+r(x, y)$. We first reconstruct $g(x, y)$. To reconstruct it, we need to use Fourier coefficients $c_{m}\left(\varphi^{(0)}\right)$, $c_{m}\left(\varphi^{(1)}\right), c_{n}\left(\psi^{(0)}\right), c_{n}\left(\psi^{(1)}\right)$, and $c_{m n}(r)$ which are stated in Section 2. From

$$
\varphi^{(0)}(x)=f(x, 0)-(f(0,0)(1-x)+f(1,0) x),
$$

we deduce that $\varphi^{(0)}(0)=\varphi^{(0)}(1)$. So

$$
\begin{aligned}
c_{m} & \left(\varphi^{(0)}\right)=\int_{0}^{1} \varphi^{(0)}(x) e^{-2 \pi i m x} \mathrm{~d} x \\
= & -\frac{1}{2 \pi i m}\left(\left.\varphi^{(0)}(x) e^{-2 \pi i m x}\right|_{x=0} ^{1}\right. \\
& \left.-\int_{0}^{1} \varphi_{x}^{(0)}(x) e^{-2 \pi i m x} \mathrm{~d} x\right)=\frac{1}{2 \pi i m} \\
& \cdot \int_{0}^{1} \varphi_{x}^{(0)}(x) e^{-2 \pi i m x} \mathrm{~d} x=-\frac{1}{(2 \pi i m)^{2}}\left(\varphi_{x}^{(0)}(1)\right. \\
& \left.-\varphi_{x}^{(0)}(0)-\int_{0}^{1} \varphi_{x^{2}}^{(0)}(x) e^{-2 \pi i m x} \mathrm{~d} x\right) .
\end{aligned}
$$

Since $f \in C^{(3,3)}\left([0,1]^{2}\right)$, we have $\varphi^{(0)} \in C^{3}([0,1])$, and so

$$
\int_{0}^{1} \varphi_{x^{2}}^{(0)}(x) e^{-2 \pi i m x} \mathrm{~d} x=O\left(\frac{1}{m}\right) .
$$

This gives an asymptotic formula as follows:

$$
c_{m}\left(\varphi^{(0)}\right)=\frac{1}{4 \pi^{2} m^{2}}\left(\varphi_{x}^{(0)}(1)-\varphi_{x}^{(0)}(0)\right)+O\left(\frac{1}{m^{3}}\right) .
$$

Differentiating (24), we get $\varphi_{x}^{(0)}(x)=f_{x}(x, 0)+f(0,0)-$ $f(1,0)$. So

$$
c_{m}\left(\varphi^{(0)}\right)=\frac{1}{4 \pi^{2} m^{2}}\left(f_{x}(1,0)-f_{x}(0,0)\right)+O\left(\frac{1}{m^{2}}\right) .
$$

Similarly, we discuss the Fourier coefficients $c_{m}\left(\varphi^{(1)}\right)$, $c_{n}\left(\psi^{(0)}\right)$, and $c_{n}\left(\psi^{(1)}\right)$. We get the following.

Theorem 1. Let $f \in C^{(3,3)}\left([0,1]^{2}\right)$ and $\varphi^{(0)}, \varphi^{(1)}, \psi^{(0)}$, and $\psi^{(1)}$ be stated in Section 2. Then, for their Fourier coefficients, one has the following asymptotic formulas:

$$
\begin{aligned}
& c_{m}\left(\varphi^{(0)}\right)=\frac{1}{4 \pi^{2} m^{2}}\left(f_{x}(1,0)-f_{x}(0,0)\right)+O\left(\frac{1}{m^{3}}\right), \\
& c_{m}\left(\varphi^{(1)}\right)=\frac{1}{4 \pi^{2} m^{2}}\left(f_{x}(1,1)-f_{x}(0,1)\right)+O\left(\frac{1}{m^{3}}\right), \\
& c_{n}\left(\psi^{(0)}\right)=\frac{1}{4 \pi^{2} n^{2}}\left(f_{y}(1,0)-f_{y}(0,0)\right)+O\left(\frac{1}{n^{3}}\right), \\
& c_{n}\left(\psi^{(1)}\right)=\frac{1}{4 \pi^{2} n^{2}}\left(f_{y}(1,1)-f_{y}(0,1)\right)+O\left(\frac{1}{n^{3}}\right) .
\end{aligned}
$$

Denote the partial sums of the Fourier series of $\varphi^{(0)}$ by $s_{N}\left(\varphi^{(0)}, x\right)$; that is,

$$
s_{N}\left(\varphi^{(0)}, x\right)=\sum_{|m|<N} c_{m}\left(\varphi^{(0)}\right) e^{2 \pi i m x} .
$$

By the Parseval identity, the mean square error of $\varphi^{(0)}$ satisfies

$$
\left\|\varphi^{(0)}-s_{N}\left(\varphi^{(0)}\right)\right\|_{2}^{2}=\sum_{|m|<N}\left|c_{m}\left(\varphi^{(0)}\right)\right|^{2} .
$$

From (37), it follows that

$$
\begin{aligned}
\left|c_{m}\left(\varphi^{(0)}\right)\right|^{2}= & \frac{1}{16 \pi^{4} m^{4}}\left|f_{x}(1,0)-f_{x}(0,0)\right|^{2} \\
& +O\left(\frac{1}{m^{5}}\right) .
\end{aligned}
$$

Noticing that $\sum_{|m|<N}\left(1 / m^{4}\right)=2 / N^{3}+O\left(1 / N^{4}\right)$, we get

$$
\begin{aligned}
\left\|\varphi^{(0)}-s_{N}\left(\varphi^{(0)}\right)\right\|_{2}^{2}= & \frac{1}{8 \pi^{4} N^{3}}\left|f_{x}(1,0)-f_{x}(0,0)\right|^{2} \\
& +O\left(\frac{1}{N^{4}}\right) .
\end{aligned}
$$

Therefore, we can reconstruct $\varphi^{(0)}$ by Fourier coefficients $c_{m}\left(\varphi^{(0)}\right)(|m|<N)$ and we obtain the asymptotic formula of the error. Again, by Theorem 1, we get

$$
\begin{aligned}
& \left\|f(x, 0)-h_{N}^{(1)}(x)\right\|_{2}^{2}=\| f(x, 0)-f(0,0)(1-x) \\
& -f(1,0) x-s_{N}\left(\varphi^{(0)} ; x\right) \|_{2}^{2}=\frac{1}{8 \pi^{4} N^{3}} \mid f_{x}(1,0) \\
& \quad-\left.f_{x}(0,0)\right|^{2}+O\left(\frac{1}{N^{4}}\right) .
\end{aligned}
$$

From this, we see that $f(x, 0)$ can be reconstructed by $f(0,0), f(1,0)$ and Fourier coefficients $c_{m}\left(\varphi^{(0)}\right)(|m|<$ $N)$, the order of the error is $1 / N^{3}$, and the coefficient is $\left(1 / 8 \pi^{4}\right)\left(f_{x}(1,0)-f_{x}(0,0)\right)^{2}$.

Similarly, for the boundary functions $f(x, 1), f(0, y)$, and $f(1, y)$, the corresponding asymptotic formulas are the following:

$$
\begin{aligned}
& \left\|f(x, 1)-h_{N}^{(2)}(x)\right\|_{2}^{2}=\| f(x, 1)-f(0,1)(1-x) \\
& \quad-f(1,1) x-s_{N}\left(\varphi^{(1)} ; x\right) \|_{2}^{2}=\frac{1}{8 \pi^{4} N^{3}} \mid f_{x}(1,1) \\
& \quad-\left.f_{x}(0,1)\right|^{2}+O\left(\frac{1}{N^{4}}\right), \\
& \left\|f(0, y)-h_{N}^{(3)}(y)\right\|_{2}^{2}=\| f(0, y)-f(0,0)(1-y) \\
& \quad-f(0,1) y-s_{N}\left(\psi^{(0)} ; y\right) \|_{2}^{2}=\frac{1}{8 \pi^{4} N^{3}} \mid f_{y}(0,1) \\
& \quad-\left.f_{y}(0,0)\right|^{2}+O\left(\frac{1}{N^{4}}\right),
\end{aligned}
$$




$$
\begin{aligned}
& \left\|f(1, y)-h_{N}^{(4)}(y)\right\|_{2}^{2}=\| f(1, y)-f(1,0)(1-y) \\
& -f(1,1) y-s_{N}\left(\psi^{(1)} ; y\right) \|_{2}^{2}=\frac{1}{8 \pi^{4} N^{3}} \mid f_{y}(1,1) \\
& \quad-\left.f_{y}(1,0)\right|^{2}+O\left(\frac{1}{N^{4}}\right) .
\end{aligned}
$$

By definitions of $g(x, y)$ and $g_{N}(x, y)$ in Section 2 , we get

$$
\begin{aligned}
&\left\|g(x, y)-g_{N}(x, y)\right\|_{2}^{2} \\
& \leq 2\left\|g_{1}(x, y)-g_{1 N}(x, y)\right\|_{2}^{2} \\
&+2\left\|g_{2}(x, y)-g_{2 N}(x, y)\right\|_{2}^{2} \\
& \leq 4\left\|f(x, 0)-h_{N}^{(1)}(x)\right\|_{2}^{2}+4\left\|f(x, 1)-h_{N}^{(2)}(x)\right\|_{2}^{2} \\
&+4\left\|f(0, y)-h_{N}^{(3)}(y)\right\|_{2}^{2} \\
&+4\left\|f(1, y)-h_{N}^{(4)}(y)\right\|_{2}^{2} .
\end{aligned}
$$

So, from the above four asymptotic formulas, we know that

$$
\left\|g(x, y)-g_{N}(x, y)\right\|_{2}^{2} \leq \frac{D}{2 \pi^{4} N^{3}}+O\left(\frac{1}{N^{4}}\right),
$$

where

$$
\begin{aligned}
D= & \left(f_{x}(1,0)-f_{x}(0,0)\right)^{2}+\left(f_{x}(1,1)-f_{x}(0,1)\right)^{2} \\
& +\left(f_{y}(0,1)-f_{y}(0,0)\right)^{2} \\
& +\left(f_{y}(1,1)-f_{y}(1,0)\right)^{2} .
\end{aligned}
$$

From this, we know that the function $g(x, y)$, defined in (18), can be reconstructed by $f(0,0), f(0,1)$ and $f(1,0)$, $f(1,1)$ and Fourier coefficients $c_{m}\left(\varphi^{(0)}\right), c_{m}\left(\varphi^{(1)}\right)(|m|<N)$ and $c_{n}\left(\psi^{(0)}\right), c_{n}\left(\psi^{(1)}\right)(|n|<N)$, and the order of error is $1 / N^{3}$.

From the reconstruction of $g(x, y)$, we know that it is a simple combination of the boundary functions of $f(x, y)$ and factors $x, y,(1-x),(1-y)$. It possesses the following important properties.

Theorem 2. Let $f$ be defined on $[0,1]^{2}$ and let $g$ be stated in Section 2. Then

$$
\begin{aligned}
& g(x, 0)=f(x, 0) \\
& g(x, 1)=f(x, 1) \\
& \qquad(0 \leq x \leq 1), \\
& g(0, y)=f(0, y) \\
& g(1, y)=f(1, y) \\
& \qquad(0 \leq y \leq 1) .
\end{aligned}
$$

That is, $g$ and $f$ have same boundary functions: $g(x, y)=$ $f(x, y)\left((x, y) \in \partial\left([0,1]^{2}\right)\right)$.
Proof. By (5), (11), and (17),

$$
\begin{aligned}
& g_{1}(x, 0)=f(x, 0) \\
& g_{2}(x, 0)=f(0,0)(1-x)+f(1,0) x, \\
& g_{3}(x, 0)=f(0,0)(1-x)+f(1,0) x .
\end{aligned}
$$

So $g(x, 0)=g_{1}(x, 0)+g_{2}(x, 0)-g_{3}(x, 0)=f(x, 0)(0 \leq$ $x \leq 1)$. Similarly, the rest equalities can be proved by the same way.

\section{Asymptotic Formula of Approximation Error for $r(x, y)$}

In this section, we will estimate the approximation errors of our proposed approximation scheme. In order to show that $r(x, y)$ in (19) can be reconstructed by few Fourier coefficients, we give the following asymptotic formula of Fourier coefficients of $r(x, y)$.

Theorem 3. Let $f \in C^{(3,3)}\left([0,1]^{2}\right)$ be defined on $[0,1]^{2}$ and let $g$ be stated as in Theorem 2, and let

$$
r(x, y)=f(x, y)-g(x, y)
$$

Then Fourier coefficients of $r(x, y)$ satisfy the following asymptotic formula:

$$
\begin{aligned}
& c_{m n}(r)=\frac{1}{16 \pi^{4} m^{2} n^{2}}\left(f_{x y}(1,1)-f_{x y}(1,0)\right. \\
& \left.-f_{x y}(0,1)+f_{x y}(0,0)\right)+O\left(\frac{1}{m^{2} n^{2}}\right)\left(\frac{1}{m}+\frac{1}{n}\right) \\
& \quad(m \neq 0, n \neq 0) .
\end{aligned}
$$

Proof. From $r(0, y)=r(1, y)=0(0 \leq y \leq 1)$, it follows that

$$
\begin{aligned}
c_{m n}(r)= & \iint_{0}^{1} r(x, y) e^{-2 \pi i(m x+n y)} \mathrm{d} x \mathrm{~d} y \\
= & \frac{1}{(2 \pi m)^{2}} \int_{0}^{1}\left(r_{x}(1, y)-r_{x}(0, y)\right) e^{-2 \pi i n y} \mathrm{~d} y \\
& -\frac{1}{(2 \pi m)^{2}} \iint_{0}^{1} r_{x^{2}}(x, y) e^{-2 \pi i(m x+n y)} \mathrm{d} x \mathrm{~d} y \\
=: & S_{m n}+T_{m n} .
\end{aligned}
$$

From $r(x, 0)=r(x, 1)=0(0 \leq x \leq 1)$, we have $r_{x}(0,0)=$ $r_{x}(0,1)=r_{x}(1,0)=r_{x}(1,1)=0$, and so

$$
\begin{aligned}
& S_{m n}=-\frac{1}{(2 \pi i m)^{2}(2 \pi i n)} \\
& \cdot \int_{0}^{1}\left(r_{x y}(1, y)-r_{x y}(0, y)\right) e^{-2 \pi i n y} \mathrm{~d} y
\end{aligned}
$$




$$
\begin{aligned}
& =\left.\frac{1}{(2 \pi i m)^{2}(2 \pi i n)^{2}}\left(r_{x y}(1, y)-r_{x y}(0, y)\right)\right|_{y=0} ^{1} \\
& -\frac{1}{(2 \pi i m)^{2}(2 \pi i n)^{2}} \\
& \cdot \int_{0}^{1}\left(r_{x y^{2}}(1, y)-r_{x y^{2}}(0, y)\right) e^{-2 \pi i n y} \mathrm{~d} y .
\end{aligned}
$$

Since $r_{x y^{2}}$ is a differentiable function of $y$, the integral value in this formula is equal to $O(1 / n)$; that is,

$$
\begin{aligned}
S_{m n}= & \left.\frac{1}{(2 \pi i m)^{2}(2 \pi i n)^{2}}\left(r_{x y}(1, y)-r_{x y}(0, y)\right)\right|_{y=0} ^{1} \\
& +O\left(\frac{1}{m^{2} n^{2}}\right) .
\end{aligned}
$$

From $r(x, y)=f(x, y)-g_{1}(x, y)-g_{2}(x, y)-g_{3}(x, y)$ and (5), (11), and (17), it can be checked that

$$
\begin{aligned}
& \left.\left(r_{x y}(1, y)-r_{x y}(0, y)\right)\right|_{y=0} ^{1} \\
& \quad=\left.\left(f_{x y}(1, y)-f_{x y}(0, y)\right)\right|_{y=0} ^{1},
\end{aligned}
$$

and so

$$
\begin{aligned}
S_{m n}= & \left.\frac{1}{(2 \pi m)^{2}(2 \pi n)^{2}}\left(f_{x y}(1, y)-f_{x y}(0, y)\right)\right|_{y=0} ^{1} \\
& +O\left(\frac{1}{m^{2} n^{2}}\right) .
\end{aligned}
$$

By $r_{x^{2}}(x, 0)=r_{x^{2}}(x, 1)=0(0 \leq x \leq 1)$, we deduce that $T_{m n}=O\left(1 / m^{2} n^{2}\right)$. From this, we get (42).

Theorem 3 shows that the decay rate of Fourier coefficients of $r$ is equivalent to $1 / m^{2} n^{2}$. The principal part of $c_{m n}(r)$ only depends on the values of $f_{x y}$ at vertices $\{0,1\}^{2}$.

Now we discuss the special case: Fourier coefficients $c_{m 0}$ and $c_{0 n}$

Since $r \in C^{(3,3)}\left([0,1]^{2}\right)$ and $r(x, y)=0((x, y) \epsilon$ $\left.\partial\left([0,1]^{2}\right)\right)$,

$$
\begin{aligned}
& \int_{0}^{1} r(x, y) e^{-2 \pi i m x} \mathrm{~d} x=\frac{1}{2 \pi m} \int_{0}^{1} r_{x}(x, y) e^{-2 \pi i m x} \mathrm{~d} x \\
& =-\frac{1}{(2 \pi m)^{2}}\left(r_{x}(1, y)-r_{x}(0, y)\right. \\
& \left.-\int_{0}^{1} r_{x^{2}}(x, y) e^{-2 \pi i m x} \mathrm{~d} x\right)=\frac{1}{(2 \pi m)^{2}}\left(r_{x}(1, y)\right. \\
& \left.-r_{x}(0, y)\right)+O\left(\frac{1}{m^{3}}\right) \quad(m \neq 0) .
\end{aligned}
$$

So

$$
\begin{aligned}
c_{m 0}(r)= & \iint_{0}^{1} r(x, y) e^{-2 \pi i m x} \mathrm{~d} x \mathrm{~d} y \\
= & \frac{1}{(2 \pi m)^{2}} \int_{0}^{1}\left(r_{x}(1, y)-r_{x}(0, y)\right) \mathrm{d} y \\
& +O\left(\frac{1}{m^{3}}\right) .
\end{aligned}
$$

Now we compute $r_{x}(1, y)-r_{x}(0, y)$.

By (5), (11), and (17) and Theorem 2, we have

$$
\begin{aligned}
g_{x}(\nu, y)= & f_{x}(\nu, 0)(1-y)+f_{x}(\nu, 1) y-f(0, y) \\
& +f(1, y)+f(0,0)(1-y)+f(0,1) y \\
& -f(1,0)(1-y)-f(1,1) y,
\end{aligned}
$$

and so

$$
\begin{aligned}
r_{x}(1, y)-r_{x}(0, y)= & f_{x}(1, y)-f_{x}(0, y)+f_{x}(0,0) \\
& -f_{x}(1,0)(1-y) \\
& +\left(f_{x}(0,1)-f_{x}(1,1)\right) y .
\end{aligned}
$$

From this and (49), it follows that $c_{m 0}(r)=A /(2 \pi m)^{2}+$ $O\left(1 / m^{3}\right)(m \neq 0)$, where

$$
\begin{aligned}
\mathrm{A}= & \int_{0}^{1}\left(f_{x}(1, y)-f_{x}(0, y)\right) \mathrm{d} y \\
& +\frac{1}{2}\left(f_{x}(0,0)-f_{x}(1,0)+f_{x}(0,1)-f_{x}(1,1)\right) .
\end{aligned}
$$

Similarly, we have $c_{0 n}(r)=B /(2 \pi n)^{2}+O\left(1 / n^{3}\right)(n \neq 0)$, where

$$
\begin{aligned}
B= & \int_{0}^{1}\left(f_{y}(x, 1)-f_{y}(x, 0)\right) \mathrm{d} x \\
& +\frac{1}{2}\left(f_{y}(0,0)-f_{y}(0,1)+f_{y}(1,0)-f_{y}(1,1)\right) .
\end{aligned}
$$

By (52), (53), and Theorem 3, it follows that

$$
\begin{aligned}
& \left|c_{m n}(r)\right|^{2}=\frac{C^{2}}{(2 \pi m)^{4}(2 \pi n)^{4}}+O\left(\frac{1}{m^{4} n^{4}}\right)\left(\frac{1}{m}+\frac{1}{n}\right) \\
& \quad(m \neq 0, n \neq 0), \\
& \left|c_{m 0}(r)\right|^{2}=\frac{A^{2}}{(2 \pi m)^{4}}+O\left(\frac{1}{m^{5}}\right) \quad(m \neq 0), \\
& \left|c_{0 n}(r)\right|^{2}=\frac{B^{2}}{(2 \pi n)^{4}}+O\left(\frac{1}{n^{5}}\right) \quad(n \neq 0),
\end{aligned}
$$

where $C=f_{x y}(1,1)-f_{x y}(1,0)-f_{x y}(0,1)+f_{x y}(0,0)$ and $A, B$ are stated in (52) and (53). 
To reconstruct $r(x, y)$ by few Fourier coefficients, we consider the hyperbolic cross truncation of the Fourier series of $r(x, y)$ :

$$
\begin{aligned}
r_{N}(x, y)= & \sum_{|m|<N} c_{m 0}(r) e^{2 \pi i m x} \\
& +\sum_{0<|n|<N} \sum_{|m| \leq N /|n|} c_{m n}(r) e^{2 \pi i(m x+n y)},
\end{aligned}
$$

and so

$$
\begin{aligned}
& r(x, y)-r_{N}(x, y) \\
& =\sum_{|m| \geq N} c_{m 0}(r) e^{2 \pi i m x}+\sum_{|n| \geq N} \sum_{m} c_{m n}(r) e^{2 \pi i(m x+n y)} \\
& \quad+\sum_{0<|n|<N} \sum_{|m| \geq N /|n|} c_{m n}(r) e^{2 \pi i(m x+n y)}
\end{aligned}
$$

By the Parseval identity, the mean square error satisfies

$$
\begin{aligned}
\| r & (x, y)-r_{N}(x, y) \|_{2}^{2} \\
= & \sum_{|m| \geq N}\left|c_{m 0}\right|^{2}+\sum_{|n| \geq N}\left|c_{n 0}\right|^{2}+\sum_{|n| \geq N} \sum_{m \neq 0}\left|c_{m n}(r)\right|^{2} \\
& +\sum_{0<|n|<N} \sum_{|m| \geq N /|n|}\left|c_{m n}(r)\right|^{2} \\
= & D_{N}^{(1)}+D_{N}^{(2)}+D_{N}^{(3)}+D_{N}^{(4)},
\end{aligned}
$$

where

$$
\begin{aligned}
& D_{N}^{(1)}=\frac{A^{2}}{(2 \pi)^{4}} \sum_{|m| \geq N} \frac{1}{m^{4}}+O\left(\frac{1}{N^{4}}\right)=\frac{A^{2}}{8 \pi^{4} N^{3}} \\
&+O\left(\frac{1}{N^{4}}\right), \\
& D_{N}^{(2)}=\frac{B^{2}}{(2 \pi)^{4}} \sum_{|n| \geq N} \frac{1}{n^{4}}+O\left(\frac{1}{N^{4}}\right)=\frac{B^{2}}{8 \pi^{4} N^{3}} \\
&+O\left(\frac{1}{N^{4}}\right), \\
& D_{N}^{(3)}=\frac{C^{2}}{(2 \pi)^{8}}\left(\sum_{|n| \geq N} \frac{1}{n^{4}}\right)\left(\sum_{m \neq 0} \frac{1}{m^{4}}\right)+O\left(\frac{1}{N^{4}}\right) \\
&=\frac{4 \gamma C^{2}}{(2 \pi)^{8} N^{3}}+O\left(\frac{1}{N^{4}}\right)\left(\gamma=\sum_{m=0}^{\infty} \frac{1}{m^{4}}\right), \\
& D_{N}^{(4)}=\frac{C^{2}}{(2 \pi)^{8}} \sum_{0<|n|<N}\left(\frac{1}{n^{4}} \sum_{|m| \geq N /|n|} \frac{1}{m^{4}}\right)+O(1) \\
& \cdot \sum_{0<|n|<N}\left(\frac{1}{N^{4}} \sum_{|m| \geq N /|n|} \frac{1}{m^{5}}\right)+O(1) \\
& \cdot \sum_{0<|n|<N}\left(\frac{1}{n^{5}} \sum_{|m| \geq N /|n|} \frac{1}{m^{4}}\right) .
\end{aligned}
$$

Noticing that $\sum_{|m| \geq N /|n|}\left(1 / m^{4}\right)=2|n|^{3} / N^{3}+O\left(n^{4} / N^{4}\right)$ and $\sum_{|m| \geq N /|n|}\left(1 / m^{5}\right)=2|n|^{4} / N^{4}+O\left(|n|^{5} / N^{5}\right)$, we deduce that

$$
\begin{aligned}
D_{N}^{(4)} & =\frac{2 C^{2}}{(2 \pi)^{8}}\left(\sum_{0<|n|<N} \frac{1}{|n|}\right) \frac{1}{N^{3}}+O\left(\frac{1}{N^{3}}\right) \\
& =\frac{4 C^{2}}{(2 \pi)^{8}}\left(\int_{1}^{N} \frac{1}{x} \mathrm{~d} x\right) \frac{1}{N^{3}}+O\left(\frac{1}{N^{3}}\right) \\
& =\frac{4 C^{2}}{(2 \pi)^{8}} \frac{\log N}{N^{3}}+O\left(\frac{1}{N^{3}}\right) .
\end{aligned}
$$

From this and (57), we obtain finally the following theorem.

Theorem 4. Suppose that $f(x, y) \in C^{(3,3)}\left([0,1]^{2}\right)$ and $r(x, y)$ be stated in (19) and $r_{N}(x, y)$ be the hyperbolic cross truncation of Fourier series of $r(x, y)$. Then the mean square error of reconstruction of $r(x, y)$ by $r_{N}(x, y)$ satisfies the following asymptotic formula:

$$
\begin{aligned}
& \left\|r(x, y)-r_{N}(x, y)\right\|_{2}^{2} \\
& =\frac{4 C^{2}}{(2 \pi)^{8}} \frac{\log N}{N^{3}}+\left(\frac{1}{8 \pi^{4}}\left(A^{2}+B^{2}\right)+\frac{\alpha C^{2}}{64 \pi^{8}}\right) \frac{1}{N^{3}} \\
& \quad+O\left(\frac{1}{N^{4}}\right),
\end{aligned}
$$

where

$$
\begin{aligned}
A= & \int_{0}^{1}\left(f_{x}(1, y)-f_{x}(0, y)\right) \mathrm{d} y \\
& +\frac{1}{2}\left(f_{x}(0,0)-f_{x}(1,0)+f_{x}(0,1)-f_{x}(1,1)\right), \\
B= & \int_{0}^{1}\left(f_{y}(x, 1)-f_{y}(x, 0)\right) \mathrm{d} x \\
& +\frac{1}{2}\left(f_{y}(0,0)-f_{y}(0,1)+f_{y}(1,0)-f_{y}(1,1)\right), \\
C= & f_{x y}(1,1)-f_{x y}(1,0)-f_{x y}(0,1)+f_{x y}(0,0), \\
\alpha= & \sum_{m=1}^{\infty} \frac{1}{m^{4}} .
\end{aligned}
$$

\section{Approximation Error of $f(x, y)$}

In this section, we will compare our improved Fourier approximation scheme with traditional ones. By (18), (19), and (23),

$$
\begin{aligned}
& \left\|f(x, y)-f_{N}(x, y)\right\|_{2}^{2} \\
& \leq 2\left\|g(x, y)-g_{N}(x, y)\right\|_{2}^{2} \\
& \quad+2\left\|r(x, y)-r_{N}(x, y)\right\|_{2}^{2} .
\end{aligned}
$$


By (57) and (60),

$$
\begin{aligned}
& \left\|f(x, y)-f_{N}(x, y)\right\|_{2}^{2} \\
& \leq \frac{8 C^{2}}{(2 \pi)^{8}} \frac{\log N}{N^{3}}+\left(\frac{A^{2}+B^{2}}{4 \pi^{4}}+\frac{\alpha C^{2}}{32 \pi^{8}}+\frac{D}{\pi^{4}}\right) \frac{1}{N^{3}} \\
& \quad+O\left(\frac{1}{N^{4}}\right),
\end{aligned}
$$

where $A, B, C$, and $\alpha$ are stated as above and $D$ is stated in (38).

To construct $f_{N}(x, y)$, data used by us are four values of $f$ at vertices on $[0,1]^{2}$ and $8 N-4$ univariate Fourier coefficients as well as $M$ bivariate Fourier coefficients $c_{m n}(|m n|<$ $N ;|m|,|n|<N)$, where

$$
\begin{aligned}
M & =4 N-1+\sum_{1 \leq|n|<N} \sum_{1 \leq|m|<N /|n|} 1 \\
& \leq 4 N-1+2 N \sum_{1 \leq|n|<N} \frac{1}{|n|} \\
& \leq 4 N-1+4 N\left(1+\int_{1}^{N} \frac{1}{t} \mathrm{~d} t\right) \\
& =8 N-1+4 N \log N .
\end{aligned}
$$

Therefore, in the reconstruction scheme, the total number of coefficients used by us is less than $16 N+4 N \log N$. From this and (63), we get

$$
\left\|f(x, y)-f_{N}(x, y)\right\|_{2}^{2}=O\left(\frac{\log ^{4} N_{d}}{N_{d}^{3}}\right),
$$

where $N_{d}$ is the number of coefficients used to construct $f_{N}$.

One the other hand, if we directly expand $f \in$ $C^{(3,3)}\left([0,1]^{2}\right)$ into Fourier series,

$$
f(x, y)=\sum_{m} \sum_{n} c_{m n}(f) e^{2 \pi i(m x+n y)},
$$

and use the partial sums of Fourier series as a approximation tool

$$
s_{N}(f ; x, y)=\sum_{|m|<N} \sum_{|n|<N} c_{m n}(f) e^{2 \pi i(m x+n y)}
$$

to reconstruct $f(x, y)$, then, from $c_{m n}(f) \sim 1 / m n$ [3-5], we deduce that

$$
\left\|f(x, y)-s_{N}(f ; x, y)\right\|_{2}^{2}=O\left(\frac{1}{N}\right) .
$$

There are $N_{s}=(2 N+1)^{2}$ Fourier coefficients in the partial sum $s_{N}(f ; x, y)$; that is,

$$
\left\|f(x, y)-s_{N}(f ; x, y)\right\|_{2}^{2}=O\left(\frac{1}{\sqrt{N_{s}}}\right) .
$$

Comparing this formula with (65), we see that the approximation scheme proposed in this paper has obvious advantage over traditional method.

\section{Conflicts of Interest}

The author declares that there are no conflicts of interest regarding the publication of this paper.

\section{Acknowledgments}

This research is partially supported by National Key Science Program no. 2015CB953602; Fundamental Research Funds for the Central Universities (Key Program) no. 105565GK; Beijing Young Talent Fund and Scientific Research Foundation for the Returned Overseas Chinese Scholars, State Education Ministry.

\section{References}

[1] E. M. Stein and G. Weiss, Introduction to Fourier Analysis on Euclidean Spaces, Princeton University Press, 1971.

[2] Z. Zhang, "Fourier analysis on trapezoids with curved sides," Indian Journal of Pure and Applied Mathematics, vol. 43, no. 5, pp. 495-520, 2012.

[3] G. G. Lorentz, M. von Golitschek, and J. Makovoz, Constructive Approximation, Advanced Problems, Springer-Verlag, Berlin, Germany, 1996.

[4] Z. Zhang, "Reconstruction of bivariate functions by sparse sine coefficients," Journal of Computational Analysis and Applications, vol. 20, no. 3, pp. 530-547, 2016.

[5] Z. Zhang and J. C. Moore, Mathematical and Physical Fundamentals of Climate Change, Elsevier, 2015.

[6] P. E. Jorgensen, "Measures in wavelet decompositions," Advances in Applied Mathematics, vol. 34, no. 3, pp. 561-590, 2005.

[7] P. E. Jorgensen, "Minimality of the data in wavelet filters," Advances in Mathematics, vol. 159, no. 2, pp. 143-228, 2001.

[8] C. K. Chui, X. C. Shen, and L. Zhong, "On Lagrange polynomial quasi-interpolation," in Topics in Polynomials of One and Several Variables and Their Applications, pp. 125-141, World Scientific, River Edge, NJ, usa, 1993.

[9] C. K. Chui and H. Diamond, "A characterization of multivariate quasi-interpolation formulas and its applications," Numerische Mathematik, vol. 57, no. 2, pp. 105-121, 1990.

[10] M.-J. Lai, “The convergence of three $L_{1}$ spline methods for scattered data interpolation and fitting," Journal of Approximation Theory, vol. 145, no. 2, pp. 196-211, 2007.

[11] M.-J. Lai, "Convex preserving scattered data interpolation using bivariate $C^{1}$ cubic splines," Journal of Computational and Applied Mathematics, vol. 119, no. 1-2, pp. 249-258, 2000.

[12] J. Shen and H. Yu, "Efficient spectral sparse grid methods and applications to high-dimensional elliptic problems," SIAM Journal on Scientific Computing, vol. 32, no. 6, pp. 3228-3250, 2010. 


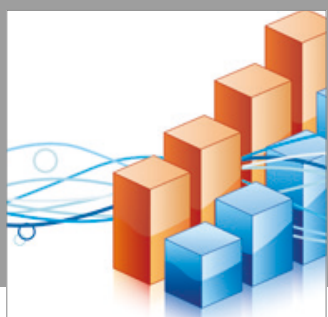

Advances in

Operations Research

vatersals

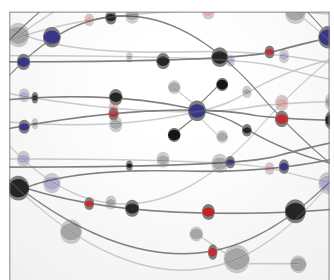

\section{The Scientific} World Journal
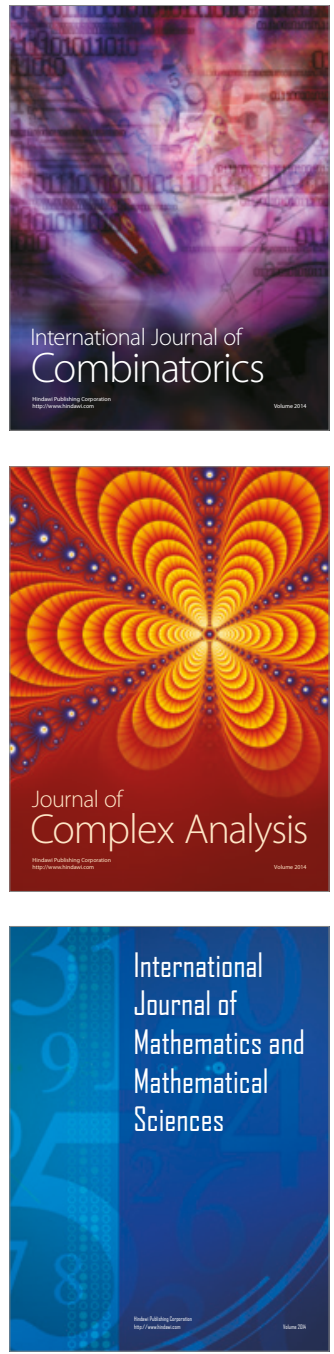
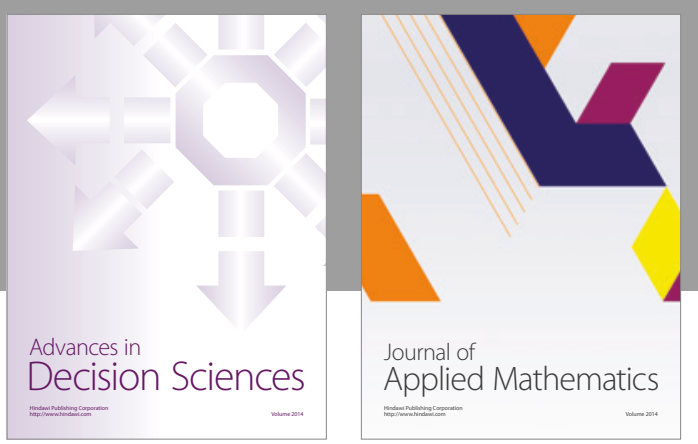

Algebra

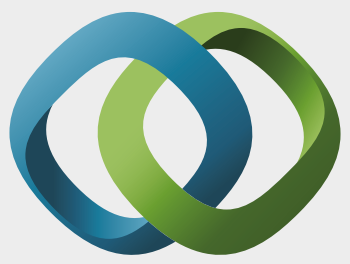

\section{Hindawi}

Submit your manuscripts at

https://www.hindawi.com
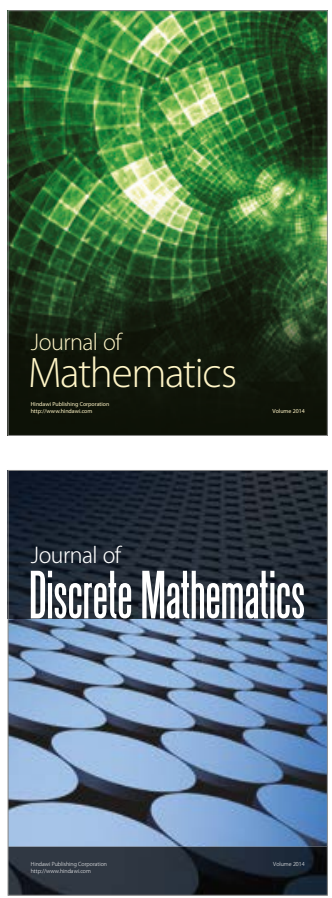

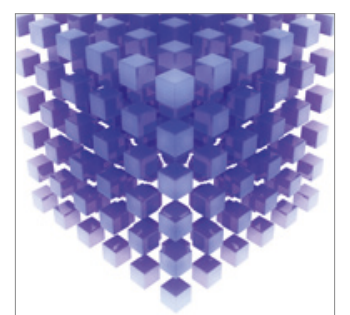

Mathematical Problems in Engineering
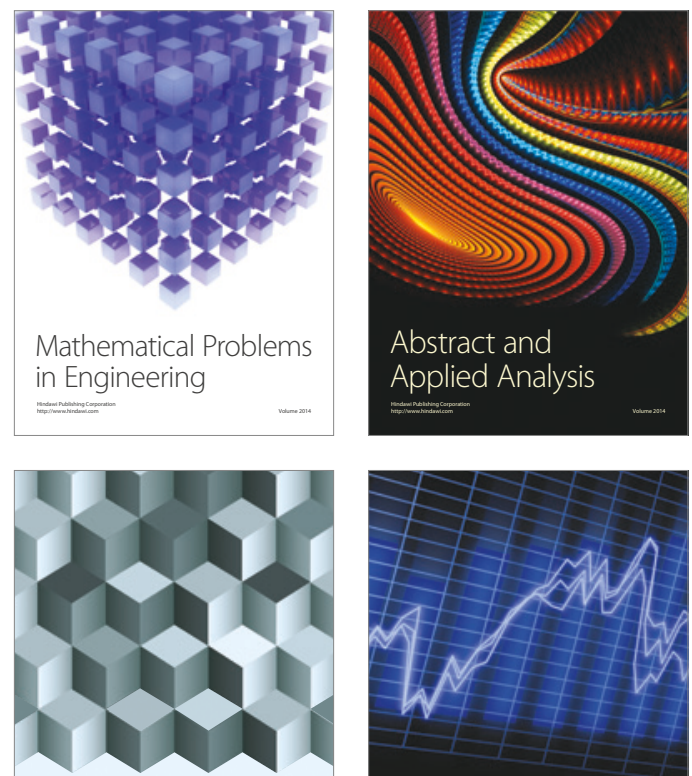

Journal of

Function Spaces

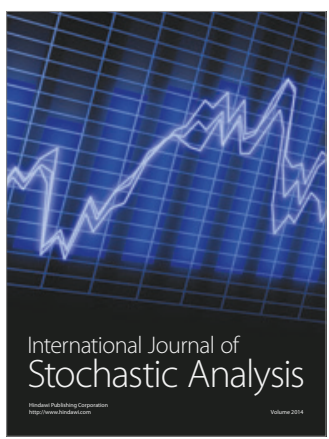

Probability and Statistics
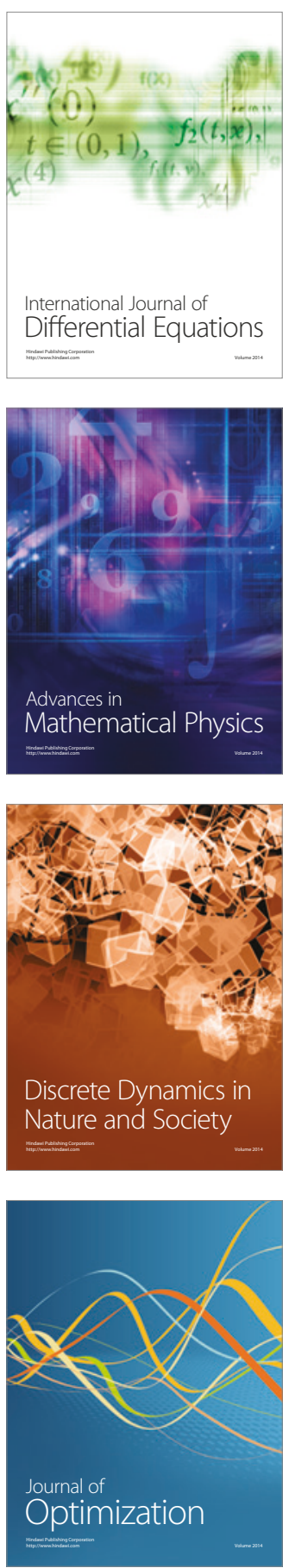